\title{
Supporting Heart Failure Patients Through Personalized Mobile Health Monitoring
}

\author{
Andreas Triantafyllidis, Carmelo Velardo, Syed \\ Ahmar Shah, Lionel Tarassenko \\ Institute of Biomedical Engineering, Department of \\ Engineering Science, University of Oxford \\ Oxford, UK
}

\author{
Tracey Chantler, Chris Paton, Kazem Rahimi \\ George Institute for Global Health, Nuffield Department \\ of Population Health, University of Oxford \\ Oxford, UK
}

\begin{abstract}
Heart failure is a common chronic condition requiring frequent attention and ongoing provision of healthcare services. In this context we present a personalized mobile-based home monitoring system aiming to support heart failure patients in daily self-monitoring of their condition. An Internet-linked tablet computer and various portable and wearable sensing devices are employed in order to monitor the patient's physiological parameters and enable healthcare professionals to review patient's status remotely. The proposed system supports the activation/deactivation of system functional components by healthcare professionals during run-time operation, the unobtrusive remote upgrade of the mobile system through a private application distribution channel, and the automatic recording of user interactions, in order to meet the patient's ongoing individualized preferences and healthcare needs. Preliminary results from an observational cohort study indicate that heart failure patients find the proposed system acceptable and consider it useful for self-monitoring their condition.
\end{abstract}

Keywords- mobile health; home monitoring; personalization; heart failure; pervasive healthcare

\section{INTRODUCTION}

Pervasive healthcare, i.e., the provision of healthcare services to individuals anytime-anywhere, has recently become the focus of the research community [1]. In this context, a plethora of health monitoring systems have been developed, in order to provide patients with access to healthcare services, reduce costs, or improve clinical outcomes [2], [3]. Sensors for monitoring a patient's physiological parameters and smart mobile computing devices, which enable the remote capture, transmission and processing of generated health data, constitute the backbone of such pervasive healthcare systems [4], [5].

Heart failure is a common chronic condition characterized by periods of physiological deterioration, often linked to worsening symptoms. This requires frequent attention from healthcare providers, which is often not possible with current episodic models of care. Mobile health technologies may therefore be particularly suited to enabling closer monitoring of the status of heart failure patients in the community, and supporting patients in the management of their condition. However, current systems have not been widely adopted by the patients mainly because of design flaws that hinder sufficient user engagement [6]. Too often the need to tailor systems to user needs or preferences is neglected, resulting in solutions that do not support effective and efficient self-management [7].

In this paper we describe a personalized mobile health system for heart failure patients, consisting of an Internetlinked tablet computer with touch-screen interfaces. Sensing devices for monitoring physiological parameters such as heart rate, blood pressure, weight, oxygen saturation $\left(\mathrm{SpO}_{2}\right)$, and activity, allow healthcare professionals to review patients' status remotely. The proposed system utilizes a range of functional and architectural features, with the aim of providing an individualized health monitoring service within the patient's home environment. More specifically, functional features of the system, besides vital sign monitoring, include the selfreporting of symptoms based on clinically-validated instruments, the review of personal readings through graphical displays, the access to educational material about heart failure self-management, and communication with healthcare professionals through text messages. Architectural features of the system include the automatic recording of user interactions, the remote update of the provided services through a private application distribution channel, and the activation/deactivation of functional components by the healthcare professionals during run-time operation. The development of this mobile system is described below. Preliminary results and initial qualitative findings of user experiences are also presented.

\section{METHODS}

\section{A. System Overview}

As seen in Fig. 1, the tablet computer-based system utilizes Bluetooth communication technology in order to communicate with a blood pressure monitor, a set of weighing scales, a pulse oximeter, and a bio-patch for continuous measurement of heart rate and activity. The monitoring data are transmitted to a backend infrastructure through Internet, enabling healthcare professionals to review the patients' health status remotely.

\section{B. Patient Interface}


Patients are enabled to: a) complete symptom diaries and take measurements, b) review personal readings using an intuitive graphical display, c) access educational material, and d) communicate with health professionals via text messages. As illustrated in Fig. 2, a linear layout is used for the completion of self-monitoring tasks, based on which patients can navigate sequentially from one task to another. A tick mark appears whenever patients successfully complete a selfmonitoring task, aiming to increase their self-confidence, keeping them motivated to continue to he tasks that follow, and potentially increasing their engagement with the system. Furthermore, detailed instructions accompanied with animated videos are displayed in order to help patients in their day-today use of the monitoring devices.

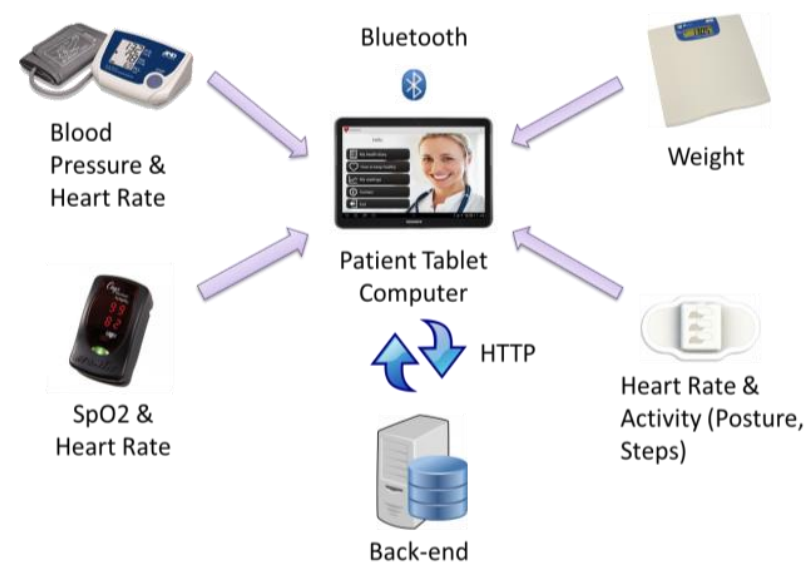

Figure 1. System overview

Symptom diaries constitute clinically validated questionnaire-based instruments in order to assess the patient's health status. Examples of such instruments include the New York Heart Association (NYHA) [8], the EuroQol (EQ-5D) [9], and the Minnesota Living With Heart Failure (MLWHF) [10] questionnaires. In addition, patients can view their personal measurements as colour graphs on the tablet screen. The provision of this type of reflection has been shown to aid towards recognising changes in health condition and adopting better health behaviours [11]. The educational material within the system includes videos about heart failure selfmanagement, aiming to further support patients. Finally, in case of a usability or healthcare problem, patients can send communication requests which are delivered via both SMS and email to the healthcare professionals, helping in the quick resolution of detected issues.

\section{Health Professional Interface}

The back-end infrastructure allows clinicians to view patient data, record notes, and send messages to the patients through a web-based application. Displayed patient data include the monitoring data as captured through the symptom diaries and from the sensing devices, as well as additional demographic and clinical information gathered automatically from National Health System (NHS) databases for each patient. Health professionals can record both health-related notes (e.g., hospitalizations, unscheduled medical visits, etc.) and notes about usability issues (e.g., inability to use a sensing device, problems with the touch-screen interfaces, etc.). The web-based application allows also clinicians to communicate the recorded notes through email to other members of the patient's care team, so as to enable more efficient patient support and problem resolution. Personalized text messages can be sent by the clinicians to the patients in order to inform them about their condition, motivate them to engage further with the system, etc.

\section{Architectural Features}

An essential feature of the system architecture is the automatic recording of user interactions. Data showing the time and duration of tasks during user navigation through the

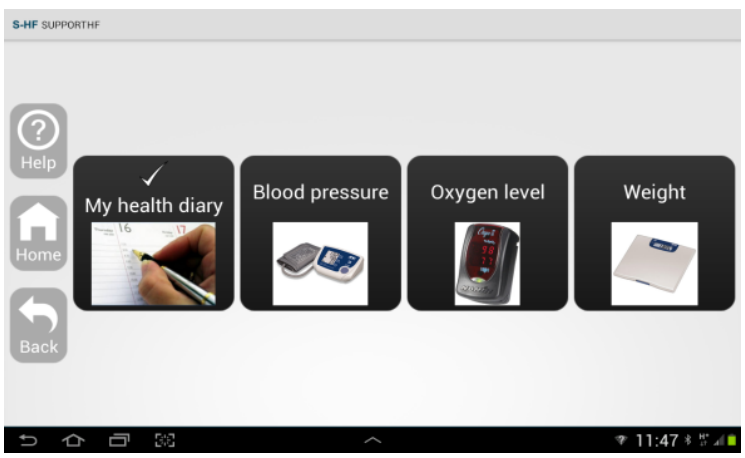

(a) Self-monitoring tasks

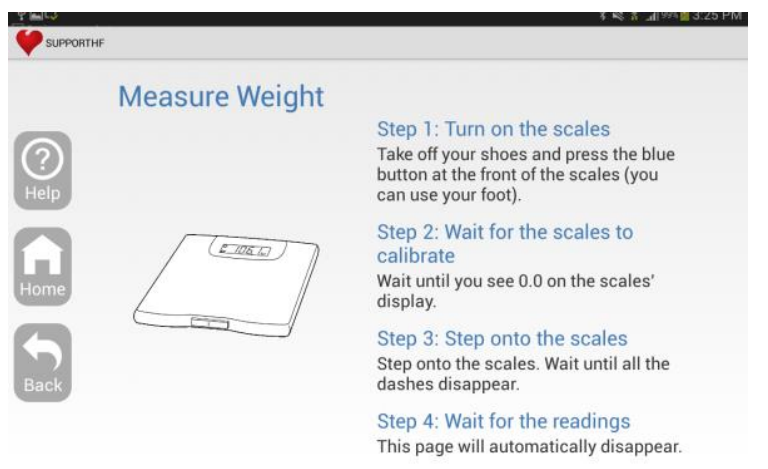

(b) Weight self-monitoring

Figure 2. Touch-screen interfaces for: (a) self-monitoring task completion, and (b) weight self-monitoring

system's functional components are stored in the back-end database. This allows the assessment of user engagement with the various system functions, the identification of patterns of use, and possibly the detection of usability issues for all or specific patients (e.g., whenever a long task duration is detected).

A private application distribution channel is used to remotely update and refine the system in an unobtrusive manner (as described more extensively in sub-section $\mathrm{E}$ below). This is currently achieved by employing Google Play Store as the enabling technology [12].

Clinicians can activate or deactivate functional components of the system during system run-time operation. As an example, clinicians can deactivate through their web- 


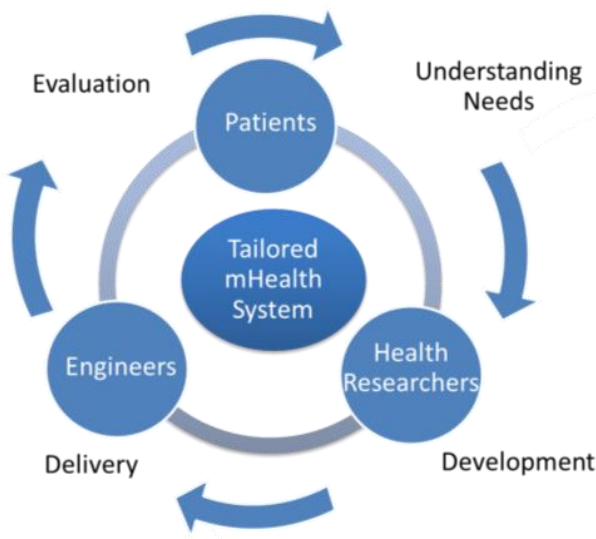

Figure 3. System design and development toward a tailored mobile health (mHealth) system

based application the self-monitoring component associated with a specific device for a particular patient, if he or she finds it difficult to use the device properly (e.g., a patient might not be able to use the pulse oximeter due to physical disability). This feature allows for a more modular and personalized approach in the delivery of health monitoring services to the patients.

\section{E. System Refinement Methodology}

We followed an iterative patient-centered approach for the ongoing development and refinement of the system involving collaboration between patients, health professionals or researchers, and software engineering experts, in order to provide services tailored to patients' preferences and healthcare needs (Fig. 3). In this context, after the initial development of the system and its use within the home environment, further system refinements took place according to: a) feedback obtained by patients during health professionals' interviews at patients' homes at regular intervals, b) the assessment of user engagement through the recorded logs of user interactions, and c) patient communication requests followed by phone interviews. The refinements of the system were in the form of mobile application updates delivered to the patient's tablet computer via the application distribution channel (i.e., Google PlayStore).

\section{RESULTS}

\section{A. System Development}

The mobile-based health monitoring system was developed using the Android programming platform [13]. Android was used because it is commonly found in smartphones and tablet computers, and also provides Application Programming Interfaces (APIs) which can deal with the restrictions found in mobile devices (e.g., small screen, limited memory size and processing capabilities). The Bluetooth protocol was used for the wireless communication with the sensing devices according to the proprietary protocol provided by the device manufacturer.

The communication between the tablet computer and the back-end infrastructure was based on $3 \mathrm{G} / 4 \mathrm{G}$ mobile data network technologies and the HTTP-based request and response model. Transport Layer Security (TLS) was utilized as the cryptographic protocol over the Internet [14]. To restrict unauthorized access, credentials were requested from health professionals in order to review patient data within their webbased application.

After patient feedback (according to our system refinement methodology), usability issues related to functional or nonfunctional features were addressed by providing remote system upgrades. Functional features were upgraded as follows: the data visualization (graphs) for patient review was improved, while instructions for the completion of symptom diaries at a time convenient for the patient were added. With respect to non-functional features, such as the data quality of the selfmonitoring data and the transmission of data to the back-end infrastructure, filtering algorithms were applied to the physiological data, while a new data synchronization mechanism for periodic communication with the back-end was introduced, to cope with intermittent mobile data network dropouts.

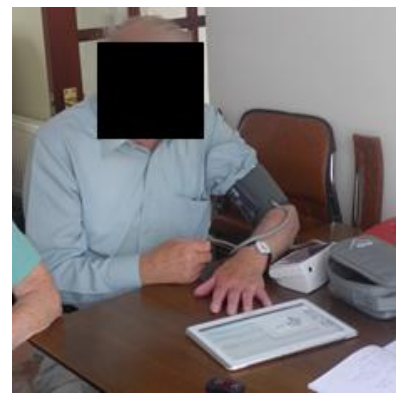

Figure 4. Patient using the proposed mobile system at home

\section{B. Preliminary Quantitative Results \& User Satisfaction}

The system was deployed during a non-interventional cohort study with heart failure patients (Fig. 4). The data collected during an initial period of 6 months from 14 patients are now reviewed here. The mean age of the patients was $74 \pm 10.34$.

In total, 1930 blood pressure, 1938 weight and 1397 oximetry self-monitoring data points were recorded. In addition, 2013 questionnaires (NYHA, EQ5D and MLWHF) were completed. Patient adherence to using the system was constantly high throughout the study and during the last week, 11 out of 14 patients (78.57\%) used it for at least 3 days.

User experiences were explored through semi-structured interviews. In this context, patients found the system particularly helpful in monitoring their condition. Two patients for example reported:

"I am doing it for my benefit as well. It is telling me how I am keeping on an ongoing basis. I use it to keep an eye on things. If I found something that was really abnormal I would check in with the GP" Participant ID 74

"OK. I thought it was to keep track of me actually. See my day to day sort of happenings, well not happenings, how I felt really" Participant ID 77. 
Other patients expressed reassurance that their health data were sent for review by health professionals. One patient specifically said:

"Anything they want me to do, they look after me pretty well, that's all I do backwards and forwards to the doctors... As long as you are getting the data...that is what matters, you see any variation, you will contact me" Participant ID 78.

\section{DISCUSSION}

We have presented a personalized mobile system for heart failure patients utilizing a tablet computer with touch-screen interfaces, and Bluetooth-enabled sensors and monitors for measuring physiological parameters. A key characteristic of the system described above was the support of architectural features, namely the automatic recording of user interactions, an application distribution channel for remote system upgrades, and remote activation or deactivation of functional components by health professionals. All of these combine to realize a mobile system adapted to patients' usability and healthcare needs.

Our preliminary results suggest that the system was found to be acceptable by patients who engaged in using it on a longterm basis, despite their age. Overall, as the qualitative results indicate, patients were satisfied by their use of the system and they found it useful for self-monitoring. Moreover, patients did not encounter any major problem in using the system, while minor usability issues were resolved after remote system upgrades, following our refinement methodology. A few patients, who faced problems in using the pulse oximeter mainly due to motor deficits, were asked to stop using it. Health professionals then remotely deactivated the pulse oximetry self-monitoring component for that patient.

Refinements took place after initial system deployment at home, to meet patient requirements and provide a sustainable system for self-monitoring. The refinements were primarily based on frequent interactions between the health researchers and the patients, so as to solicit useful feedback and improve the system. However, designers of future mobile health systems should also consider that such interactions may introduce a significant cost to the development of such systems and work overload to the health professionals. Furthermore, for major system upgrades including added functionality, formal usability studies conducted with patients are likely to be necessary [15], [16].

Our future work includes the integration of the system with Electronic Health Records (EHRs), followed by the provision of clinical decision support for drug management and titration. Furthermore, large-scale studies are required in order to assess the clinical outcomes and cost-effectiveness of the mobile health system.

In conclusion, we described a mobile health monitoring system for heart failure self-monitoring, presenting a range of functional and architectural features which can be leveraged by designers of future systems toward the construction of feasible, effective, and personalized health monitoring interventions.

\section{ACKNOWLEDGEMENTS}

The work described in this paper was supported by the NIHR Biomedical Research Centre programme and the George Institute for Global Health. Dr Kazem Rahimi is funded by an NIHR Career Development Fellowship.

Educational material within the described intervention was extracted from heartfailurematters.org and was reproduced with kind permission of the European Society of Cardiology and the British Heart Foundation. We are also grateful to the Health Experiences Research Group, Department of Primary Care Health Sciences, University of Oxford for sharing interview extracts with us from their study of heart failure, which are also available on the www.healthtalkonline.org website.

We would like to thank our study participants for their support of our research.

\section{REFERENCES}

[1] U. Varshney, "Pervasive Healthcare and Wireless Health Monitoring," Mob. Networks Appl., vol. 12, no. 2-3, pp. 113-127, Jul. 2007.

[2] D. Dorr, L. M. Bonner, A. N. Cohen, R. S. Shoai, R. Perrin, E. Chaney, and A. S. Young, "Informatics systems to promote improved care for chronic illness: a literature review.," J. Am. Med. Inform. Assoc., vol. 14, no. 2, pp. 156-63, 2007.

[3] K. Wac, "Smartphone as a personal, pervasive health informatics services platform: literature review," Yearb. Med. Inform., vol. 7, no. 1, pp. 83-93, 2012.

[4] A. Triantafyllidis, V. Koutkias, I. Chouvarda, and N. Maglaveras, "A Pervasive Health System Integrating Patient Monitoring, Status Logging and Social Sharing.," IEEE Trans. Inf. Technol. Biomed., Nov. 2012.

[5] A. Farmer, O. Gibson, P. Hayton, K. Bryden, C. Dudley, A. Neil, and L. Tarassenko, "A real-time, mobile phone-based telemedicine system to support young adults with type 1 diabetes.," Inform. Prim. Care, vol. 13, no. 3, pp. 171-7, Jan. 2005.

[6] S. I. Chaudhry, J. A. Mattera, J. P. Curtis, J. A. Spertus, J. Herrin, Z. Lin, C. O. Phillips, B. V Hodshon, L. S. Cooper, and H. M. Krumholz, "Telemonitoring in patients with heart failure.," N. Engl. J. Med., vol. 363, no. 24, pp. 2301-9, Dec. 2010.

[7] B. Maric, A. Kaan, A. Ignaszewski, and S. A. Lear, "A systematic review of telemonitoring technologies in heart failure.," Eur. J. Heart Fail., vol. 11, no. 5, pp. 506-17, May 2009.

[8] R. Holland, B. Rechel, K. Stepien, I. Harvey, and I. Brooksby, "Patients' self-assessed functional status in heart failure by New York Heart Association class: a prognostic predictor of hospitalizations, quality of life and death.," J. Card. Fail., vol. 16, no. 2, pp. 150-6, Feb. 2010.

[9] B. Schweikert, H. Hahmann, and R. Leidl, "Validation of the EuroQol questionnaire in cardiac rehabilitation.," Heart, vol. 92, no. 1, pp. 62-7, Jan. 2006.

[10] T. S. Rector and J. N. Cohn, "Assessment of patient outcome with the Minnesota Living with Heart Failure questionnaire: Reliability and validity during a randomized, double-blind, placebo-controlled trial of pimobendan," Am. Heart J., vol. 124, no. 4, pp. 1017-1025, Oct. 1992.

[11] P. Klasnja and W. Pratt, "Healthcare in the pocket: mapping the space of mobile-phone health interventions.," J. Biomed. Inform., vol. 45, no. 1, pp. 184-98, Feb. 2012.

[12] M. Butler, "Android: Changing the Mobile Landscape," IEEE Pervasive Comput., vol. 10, no. 1, pp. 4-7, Jan. 2011.

[13] R. Rogers, J. Lombardo, Z. Mednieks, and B. Meike, "Android Application Development: Programming with the Google SDK," May 2009.

[14] E. Rescorla, "HTTP Over TLS," May 2000.

[15] J. Nielsen, Usability Engineering. Morgan Kaufmann, 1993.

[16] T.-Y. Lai, "Iterative refinement of a tailored system for self-care management of depressive symptoms in people living with HIV/AIDS through heuristic evaluation and end user testing.," Int. J. Med. Inform., vol. 76 Suppl 2, pp. S317-24, Oct. 2007. 